\title{
Siringomielia "idiopática": a propósito de un caso
}

\author{
A. Horcajadas; A. Román A; G. Olivares; E. Saura; A. Jorques; B. Iáñez; J. Pastor* y J.M. Martín
}

Servicios de Neurocirugía y *Radiología (Sección de Neurorradiología). Hospital Virgen de las Nieves. Granada.

\section{Resumen}

La siringomielia se define como una cavidad intramedular llena de líquido cefalorraquídeo (LCR). En la etiología de toda cavidad siringomiélica subyace una alteración en la circulación del LCR de diversa índole. Hablamos de formas "idiopáticas" cuando no se identifican mecanismos causales de la misma. El uso de procedimientos derivativos ha sido ampliamente practicado pero el resultado de los mismos, especialmente a largo plazo, no es favorable y asocian un índice de complicaciones importante. Otras modalidades de tratamiento pretenden restaurar la circulación del LCR, eliminando así el mecanismo causal de la cavidad. Los resultados con este tipo de tratamiento son mejores a la luz de la literatura publicada. Un adecuado estudio radiológico permite identificar el espacio problema y planear eficazmente el abordaje.

Se presenta un caso de siringomielia idiopática tratado mediante disección de bridas aracnoideas y plastia dural con buena respuesta clínica y radiológica así como se revisa la literatura publicada en torno a esta modalidad de siringomielia y los distintos abordajes terapéuticos.

PALABRAS CLAVE: Siringomielia. Idiopática. Aracnoiditis. Derivación. Brida aracnoidea.

"Idiopathic" syringomyelia: report of a case

\section{Summary}

Syringomyelia is the condition in which cavities fulfilled with CSF are found within spinal cord. Syringes are caused by obstructions of CSF pathways of different causes. If we can not find a cause responsible for the blockage, we called it "idiopathic" syringomyelia. Drainage procedures have been widely used but results, especially long-term results, are not favourable and

Recibido: 29-12-06. Aceptado: 2-02-08 complication rate is high. Some authors prefer to restore CSF circulation instead to drain the syrinx. Results of this treatment modality are better in literature than drainage procedures. Adequate radiological studies help to identify problem location and to plan the approach.

A case of "idiopathic" syringomyelia is presented. Treatment consisted on arachnoids webs dissection and dural plastia. Clinical and radiological response was good. Literature concerned about this kind of syringomyelia and treatment modalities are reviewed.

KEY WORDS: Syringomyelia. Idiopathic. Arachnoiditis. Shunt. Arachnoid web.

\section{Introducción}

La siringomielia se define como una cavidad intramedular que contiene líquido cefalorraquídeo (LCR). La génesis de esta entidad es diversa, pudiendo dividirse en tres grupos: cavidades debidas a alteraciones de la circulación del LCR, las derivadas de fenómenos atróficos medulares y las secundarias a neoplasias. Entre las siringomielias relacionadas con alteraciones en la circulación del LCR se habla de siringomielia comunicante, cuando la obstrucción al flujo de LCR se sitúa en la salida del mismo del IV ventrículo, por lo que la cavidad intramedular es continuación de una dilatación generalizada del sistema ventricular, actuando como un "V ventrículo", y de siringomielia no comunicante cuando la obstrucción es a nivel o por debajo del foramen magno. En este último grupo se sitúa las cavidades secundarias a la malformación de Chiari, postraumática o postmeningítica entre otras ${ }^{12}$. La siringomielia idiopática sería aquélla en la que no se puede identificar un fenómeno causante de la misma ${ }^{11}$.

Se trata de una entidad de manejo difícil, especialmente los casos no asociados a malformación de Chiari, existiendo notable controversia en la literatura acerca del mejor abordaje de la misma. En el presente trabajo se presenta un caso de siringomielia idiopática tratado con éxito y se revisa la literatura en torno al tema, especialmente la rela- 


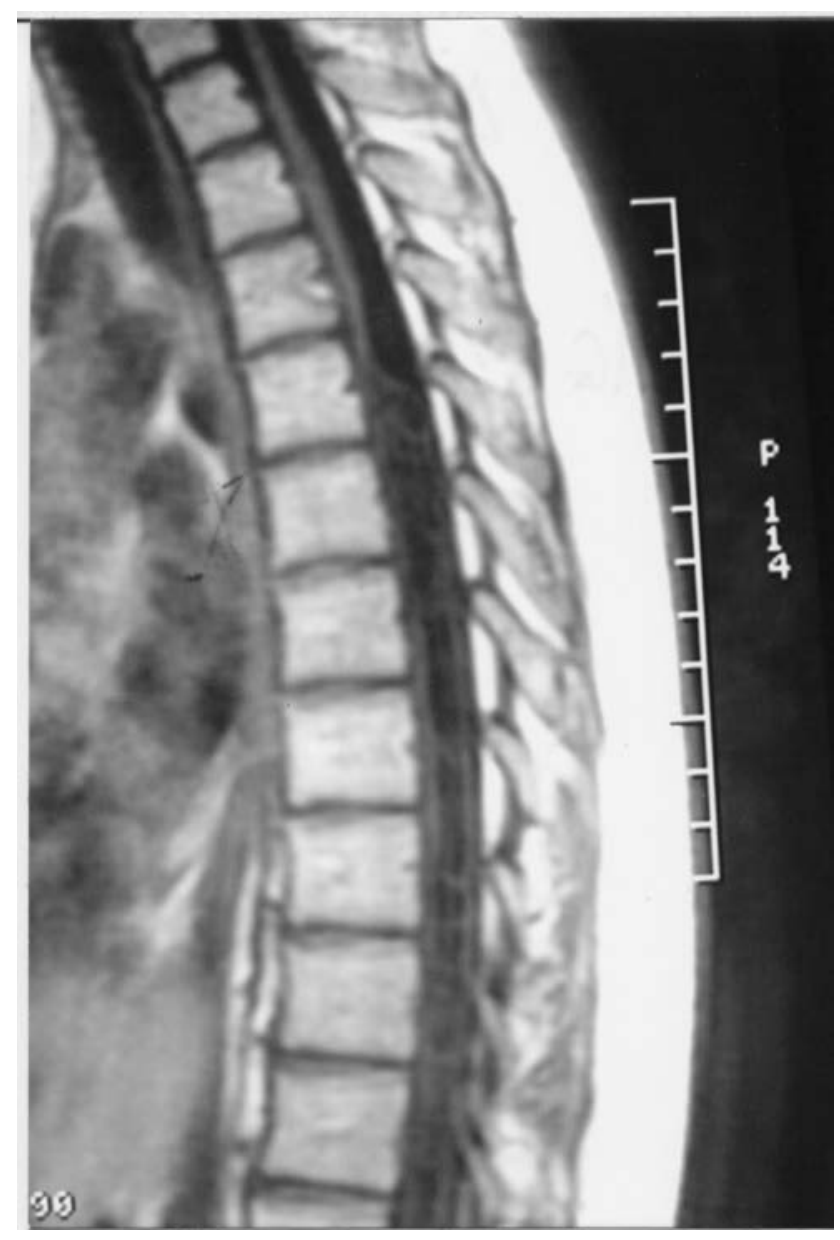

Figura 1. RMN T1 sagital en donde se aprecia un abrupto ensanchamiento del cordón medular a nivel T5-T6 con desarrollo de una cavidad siringomiélica multitabicada por debajo de dicho nivel.

cionada con las modalidades de tratamiento.

\section{Caso clínico}

Paciente mujer de 19 años sin antecedentes de interés que comienza con molestias álgicas en miembros inferiores, especialmente a nivel de rodillas. Posteriormente empieza a notar acorchamiento de miembro inferior izquierdo de inicio distal con ascenso progresivo hasta alcanzar tercio medio de muslo. La progresión de la alteración sensitiva se acompaña de la instauración de fenómenos disestésicos y sensación de pérdida de fuerza en el pie izquierdo.

En la exploración se aprecia una hemihipoestesia con nivel T4 en el lado izquierdo, hiperreflexia en miembros inferiores de predominio derecho con clonus aquíleo y Babinski bilateral así como Romberg positivo.

El estudio neurofisiológico de miembros inferiores puso de manifiesto afectación de los potenciales evocados

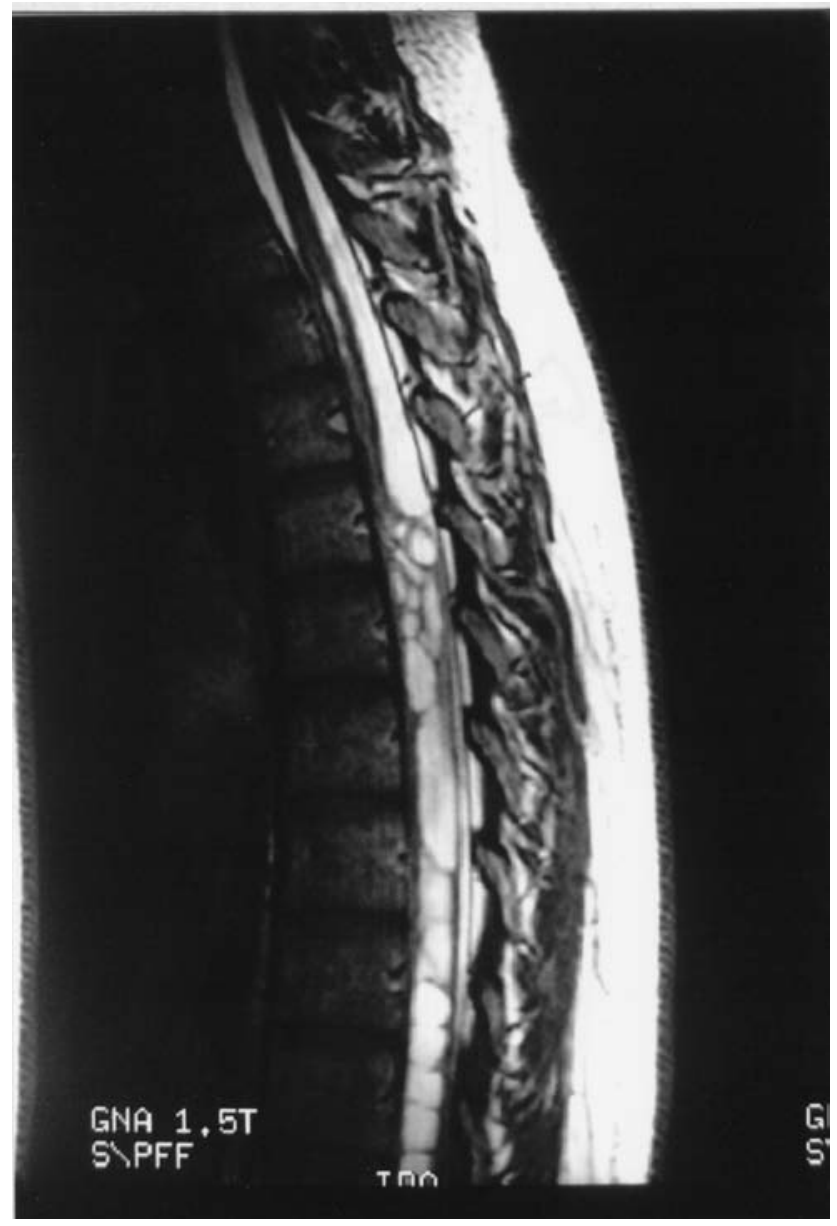

Figura 2. RMN sagital T2 donde es posible apreciar mejor la presencia de múltiples cavidades intramedulares y la aparente adhesión del cordón medular a la superficie dorsal de la duramadre. El bloqueo del espacio subaracnoideo es total.

en dicho nivel y discreta afectación de los motores.

En RMN (Figs. 1 y 2) se observa una cavidad siringomiélica que se inicia a la altura del disco interver-tebral T2T3 y que caudalmente continúa de forma muy irregular con múltiples cavidades y tabicaciones coincidentes con los niveles T5 y T6. En segmentos más inferiores se observan tabicaciones trasversales que le confieren un aspecto haustrado. No se observan anomalías de la unión cráneo cervical o áreas de captación patológica asociadas que sugieran etiología tumoral. La superficie dorsal medular a la altura de T5 se adhiere abruptamente al saco tecal, lo que sugiere un fenómeno de aracnoiditis a ese nivel.

Es intervenida quirúrgicamente a través de una laminectomía dorsal T2-T5 con control neurofisiológico intraoperatorio. En el acto quirúrgico se aprecia una banda densa de aracnoides al nivel radiológico descrito a partir de la cual la médula se encuentra ensanchada. Se procede a la 


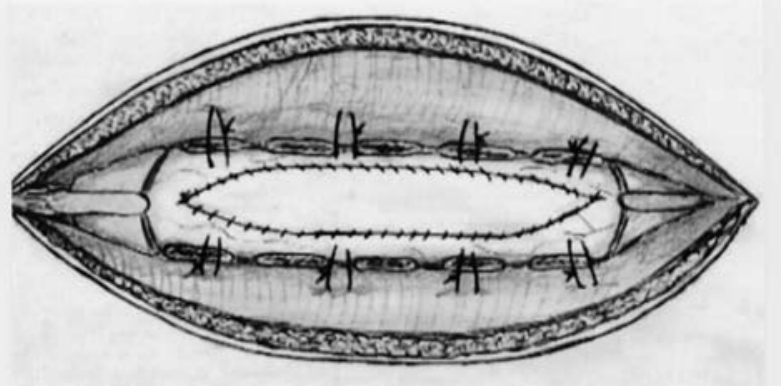

Figura 3. Dibujo esquemático del procedimiento quirúrgico de cierre efectuado, una vez liberadas las bridas aracnoideas, consistente en plastia dural y puntos de elevación de duramadre.

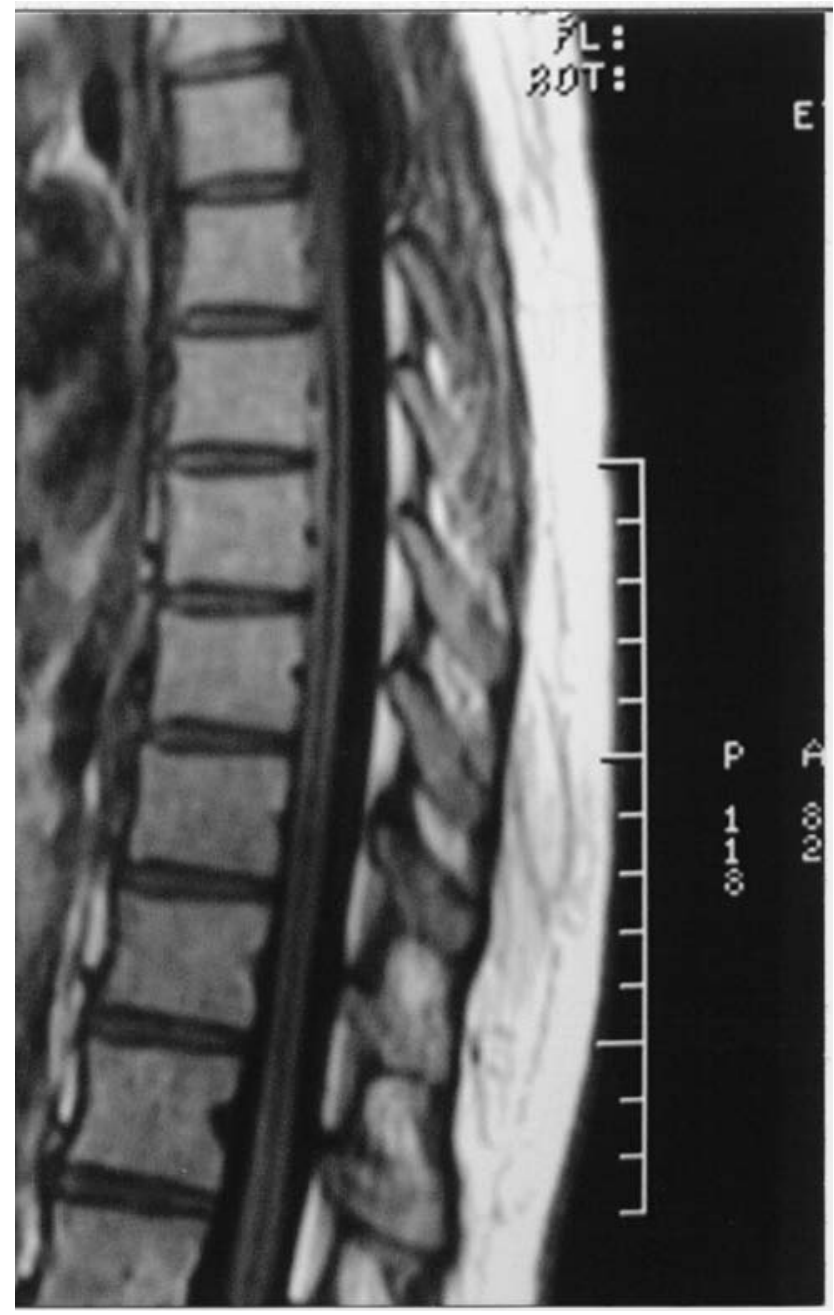

Figura 4. RMN sagital T1 de control donde es posible apreciar el colapso de la cavidad siringomiélica y la patencia

resección de dicha banda y a la colocación de un parche de duramadre que se eleva mediante puntos sueltos de sutura a las masas musculares circundantes, con el fin de mejorar

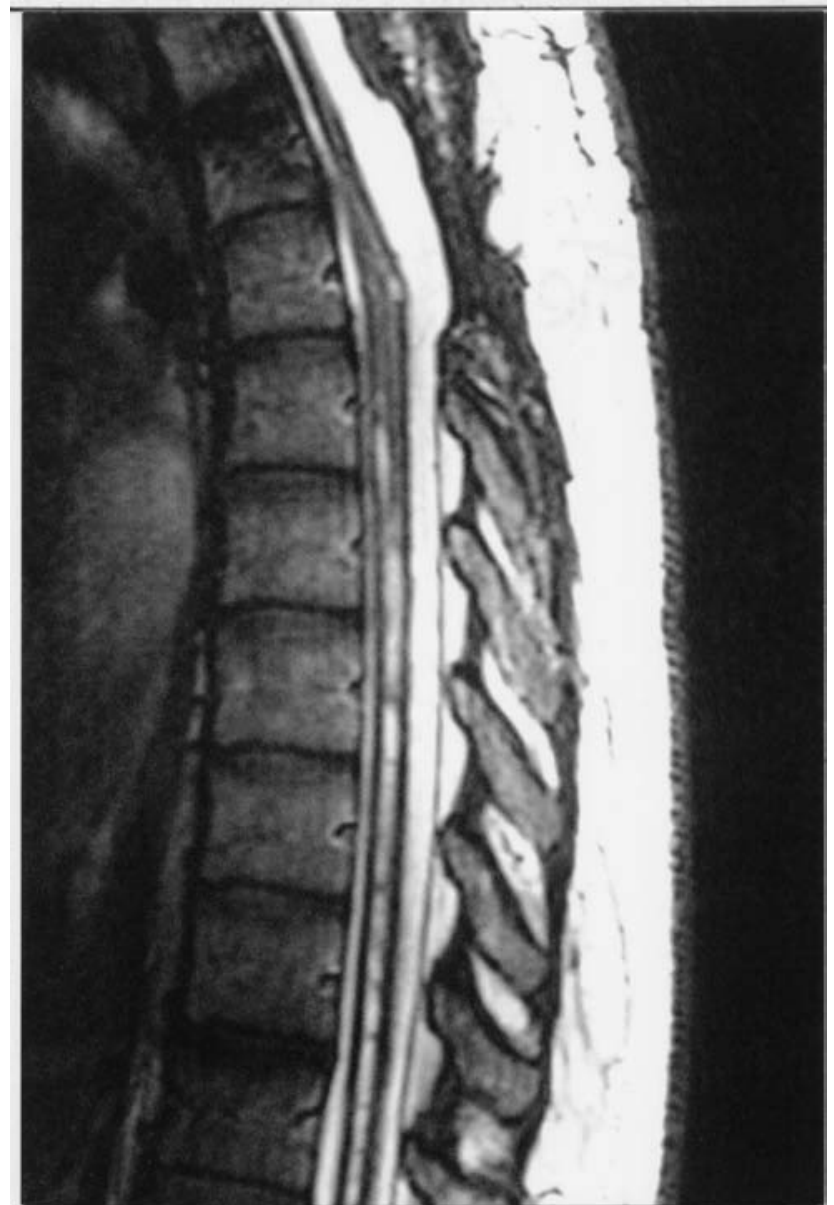

Figura 5. RMN sagital en secuencia Fiesta, más adecuada para evaluar el LCR, donde se evidencia los hallazgos descritos en la figura 4 con mayor claridad. Se aprecia cómo la cavidad siringomiélica a nivel medular se ha reducido de forma muy notable.

en lo posible la circulación del LCR a ese nivel (Fig. 3). La evolución postoperatoria fue satisfactoria y el diagnóstico anatomopatológico del tejido extirpado fue informado como paquimeningitis hipertrófica en estadio de esclerosis.

Ocho meses después de la intervención quirúrgica la paciente había mejorado notablemente, especialmente del cuadro de inestabilidad y de la pérdida de fuerza. Persistían molestias álgicas ocasionales en pierna izquierda con una frecuencia decreciente. El EMG evidenció recuperación de los potenciales motores y evocados en miembros inferiores y la RMN de control mostró la resolución de la cavidad siringomiélica por debajo del nivel intervenido, así como la patencia del espacio subaracnoideo y la efectividad de los puntos de elevación durales (Figs. 4 y 5).

\section{Discusión}

La causa más frecuente de siringomielia es la lesión 
estructural a nivel del foramen magno, con herniación de las amígdalas cerebelosas ${ }^{20}$. La segunda causa más frecuente es la postraumática. En ambos casos, la génesis, el desarrollo y el mantenimiento de la cavidad intramedular es debida al bloqueo del espacio subaracnoideo, al generarse compartimentos con distintas presiones lo que provoca que el LCR penetre en el interior de la médula a través de los espacios perivasculares por mecanismos aún no bien aclarados.

A nivel puramente espinal, el bloqueo del espacio subaracnoideo puede producirse por lesiones extradurales, como fracturas vertebrales, hernias discales o tumores de esta localización, o bien por lesiones intradurales, como las aracnoiditis postquirúrgicas, postraumáticas o postmeningíticas o tumores intradurales-extramedulares ${ }^{3}$.

El tratamiento de la siringomielia no asociada a malformación de Chiari ha sido objeto de amplia controversia, especialmente aquéllos relacionados con fenómenos de aracnoiditis.

Los casos relacionados con lesiones tumorales (intra o extradurales) o hernias discales no plantean problemas de manejo siendo la primera opción la exéresis del proceso expansivo o el disco lo que suele resolver la cavidad siringomiélica ${ }^{3,9,21}$. De modo análogo, aquellos cuadros de siringomielia postraumática secundaria a compresión medular de causa vertebral también responden a la corrección de la deformidad, siempre y cuando se restaure el espacio subaracnoideo ${ }^{6}$.

Las siringomielias secundarias a obstrucciones de la circulación de LCR de causa infecciosa o inflamatoria son las de más difícil manejo. Clásicamente se ha abogado por la colocación de derivaciones desde el quiste a diversas cavidades: espacios subaracnoideo, peritoneo o pleura. Las ventajas de este procedimiento son la inmediatez del colapso de la cavidad y la simplicidad del tratamiento. Los inconvenientes son el riego de añadir déficit neurológico por la mielotomía y la dificultad de tratar cavidades septadas. Las complicaciones incluyen obstrucciones de la derivación, hipotensiones de LCR, anclaje medular por el drenaje, infecciones y dislocación de la derivación, y el índice de las mismas puede llegar hasta el 50\%,2,3, requiriendo a menudo numerosas intervenciones por la recurrencia del quiste. Los resultados a largo plazo en algunas series tampoco son alentadores con tan solo alrededor del $50 \%$ de mejoría mantenida ${ }^{16}$. No obstante, en la literatura, especialmente en la de hace algunos años, pueden encontrarse series de pacientes tratados mediante derivaciones siringo-subaracnoideas o siringo-peritoneales con buenos resultados si bien es preciso señalar que algunas de ellas están compuestas fundamentalmente por pacientes con malformación de Chiari, cuyo pronóstico es sensiblemente mejor que otras formas de siringomielia.

Otros alternativa terapéutica es la liberación de las adherencias aracnoideas y la restauración de la circulación del LCR por el espacio subaracnoideo lo que eliminaría la causa de la formación y desarrollo de la cavidad siringomiélica ${ }^{3,5,10,13}$.

En este apartado merece la pena destacar, por su extensa serie, el trabajo de Klekamp et $\mathrm{a}{ }^{10}{ }^{10}$ sobre 107 pacientes con siringomielia no relacionada con malformación de Chiari ni tumor espinal. De esta serie, 52 pacientes padecían siringomielia postraumática y 55 pacientes siringomielia de causa inflamatoria (meningitis, hemorragia subaracnoidea, cirugía, anestesia epidural, osteomielitis, espondilodiscitis e idiopática). En un primer período el tratamiento consistió en derivación del quiste a espacios subaracnoideo, peritoneo o pleura, lo que se llevó a cabo en 61 ocasiones. Posteriormente el tratamiento pasó a ser disección y extirpación de la zona de aracnoiditis asociado en ocasiones a plastia dural con el objetivo de restablecer la libre circulación de LCR, procedimiento que se llevó a cabo en 51 ocasiones. En 7 de estos pacientes se asoció además una derivación del quiste. En los pacientes tratados mediante derivación sólo se consiguió la estabilización clínica del cuadro en el 3\% de los pacientes (porcentaje de recurrencia del 97\%), siendo los resultados ligeramente mejores en los de origen postraumático, con una morbilidad asociada a la mielotomía del $8 \%$. Radiológicamente un $49 \%$ de los quistes recurrían, un $6 \%$ no mostraban cambios y un $45 \%$ permanecían colapsados. Los resultados en los pacientes tratados con disección de la aracnoiditis y plastia dural eran sensiblemente mejores, distinguiendo entre aquéllos con aracnoiditis focal en los que se obtenían un $83 \%$ de estabilización clínica con un $4 \%$ de morbilidad y los que presentaban aracnoiditis extensa, en los que la estabilización clínica sólo alcanzaba el $17 \%$. La recurrencia en estos pacientes era mayor en los de causa traumática y si no se colocaba plastia dural. Radiológicamente un $23 \%$ de los quistes recurrían, un $12 \%$ no cambiaban y un $65 \%$ disminuían en el grupo de aracnoiditis focal y un $50 \%$ se reexpandían, un $25 \%$ permanecían y un $25 \%$ se colapsaban en el grupo de aracnoiditis extensa (Tabla 1).

La siringomielia idiopática podría definirse como aquella siringomielia no comunicante y no relacionada con malformación de Chiari en la que no se puede identificar una causa traumática, inflamatoria, infecciosa o tumoral a nivel espinal que la haya desencadenado. En la serie anteriormente descrita, en un $24 \%$ de los pacientes no se identificaba la causa de la aracnoiditis ${ }^{10}$. Distintos trabajos han evidenciado la presencia de cavidades siringomiélicas asociadas a bandas de aracnoiditis focales sin evidencia de antecedentes infecciosos, traumáticos o inflamatorios ${ }^{4,5,7,11 \text {, }}$ ${ }^{13}$. Se postulan dos orígenes para estas bandas: derivadas del septum posticum, septo presente entre la superficie dorsal de la médula y la duramadre, o de granulaciones aracnoideas atrapadas a ese nivel ${ }^{5,11}$. La presencia de estas 
Tabla 1

Resultados clínicos y radiológicos según procedimiento (J Kek/amp et al en J. Neurosurg 86:233-240, 1997)

\begin{tabular}{|l|c|c|c|c|}
\hline \multicolumn{2}{|c|}{} & \multirow{2}{*}{ Shunt } & \multicolumn{2}{c|}{ Disección } \\
\cline { 3 - 5 } & & & Aracnoiditis focal & \multicolumn{2}{c|}{ Aracnoiditis extensa } \\
\hline \multirow{3}{*}{ Clínica } & Estabilización & $3 \%$ & $83 \%$ & $17 \%$ \\
\cline { 2 - 5 } & Morbilidad & $8 \%$ & $4 \%$ & \\
\hline \multirow{3}{*}{ Radiología } & Reducción & $45 \%$ & $65 \%$ & $25 \%$ \\
\cline { 2 - 5 } & No cambio & $6 \%$ & $12 \%$ & $25 \%$ \\
\cline { 2 - 5 } & Recurrencia & $49 \%$ & $23 \%$ & $50 \%$ \\
\hline
\end{tabular}

bandas aracnoideas alteraría la circulación del LCR por el espacio subaracnoideo induciendo la formación y desarrollo de la cavidad siringomiélica. El diagnóstico en todos estos casos se realizó mediante RMN, que mostraba una adherencia más o menos brusca del cordón medular a la superficie dorsal de la duramadre, con desarrollo de cavidad siringomiélica a ese nivel con extensión craneal, caudal o ambas. El tratamiento mediante la disección del tejido aracnoideo, asociado o no a plastia dural y/o derivación del quiste, muestra un elevado porcentaje de mejoría en los trabajos revisados, que se sitúa en torno al 80\%. Algunos autores postulan la inyección de índigo carmín en el espacio subaracnoideo por encima de la banda aracnoidea mediante punción del mismo a través de una aguja del $n^{\circ}$ 27 en el acto quirúrgico a fin de identificar con precisión el stop de dicho líquido y adecuar la resección de la banda aracnoidea ${ }^{3}$.

El caso presentado puede encuadrarse dentro de lo que se ha denominado como siringomielia idiopática, al no existir entre los antecedentes de la paciente enfermedades infecciosas, traumatismos raquimedulares o intervenciones quirúrgicas previas. El estudio cuidadoso de la RMN permite detectar un abrupto cambio de dirección del cordón medular con adhesión a la superficie dorsal de la duramadre a nivel T5 y desarrollo de una siringomielia tabicada por debajo de dicho nivel. Un adecuado estudio radiológico es por lo tanto de vital importancia para planear el tratamiento quirúrgico. En el caso presentado la presencia de múltiples tabicaciones intraquísticas desaconsejaba cualquier intento de derivación de la cavidad. De cualquier modo el objetivo terapéutico planteado era la restauración de la libre circulación de LCR. La RMN permitió centrar la laminectomía en el nivel afecto, permitiendo la exéresis del tejido patológico y la ampliación del espacio subaracnoideo mediante plastia dural y puntos de elevación de la misma. La intervención quirúrgica condujo a la resolución de la cavidad siringomiélica en un plazo breve de tiempo, siendo patente en la RMN de control la permeabilidad del espacio subaracnoideo. La resolución radiológica del quiste se acompañó de una evidente mejoría clínica.

\section{Conclusión}

El manejo de la siringomielia secundaria debe encaminarse a la restauración de la circulación del LCR antes que a procedimientos de derivación de la cavidad siringomiélica. El índice de complicaciones y los pobres resultados a largo plazo de los procedimientos derivativos deberían desaconsejar esta opción terapéutica. En las siringomielias secundarias a fenómenos de aracnoiditis, los resultados de la disección de bridas aracnoideas y restauración del espacio subaracnoideo se relacionan con la extensión de la aracnoiditis y la etiología de la misma. Aquellos casos idiopáticos, en los que la zona de adhesión aracnoidea suele ser focal, tienen un índice de buenos resultados que se sitúa en torno al $80 \%$. Por tanto, la resección de bridas aracnoideas complementado con la aplicación de parches de duramadre y puntos de elevación durales que contribuyen a la restauración de la circulación del LCR por el espacio subaracnoideo, debería ser la técnica de elección.

\section{Bibliografía}

1. Alvis, C., Cerisoli, L.: Long-term results of the surgical treatment of syringohidromyelia. Acta Neurochir 1984; 71: 133-140.

2. Batzdorf, U., Klekamp, J., Johnson, J.P.: A critical appraisal of syrinx cavity shunting procedures. J Neurosurg 
1998; 89: 382-388.

3. Batzdorf, U.: Primary spinal syringomyelia: a personal perspective. Neurosurg Focus 2000; 8: Article 7, 1-4.

4. Bogdanov, E.I., Heiss, J.D., Mendelevich, E.G., Mykhaylov, I.M., Haass, A.: Clinical and neuroimaging features of "idiopathic" syringomyelia. Neurology 2004; 62: 791-794.

5. Bruneau, M., Duprez, T., Rommel, D., Raftopoulos, C.: Treatment of siryngomyelia associated with idiopathic arachnoid malformation disclosed by preoperative MRI. Surg Neurol 2004; 62: 552-555.

6. Holly, L.T., Johnston, P., Masciopinto, J.E., Batzdorf, U.: Treatment of posttraumatic syringomyelia with extradural decompressive surgery. Neurosurg Focus 2000; 8: Article 3, $1-6$.

7. Inoue, Y., Nemoto, Y., Ohata, K., et al: Siringomielia associated with adhesive spinal arachnoditis: MRI. Neurorradiology 2001; 43: 325-330.

8. Iwasaki, Y., Koyanagi, I., Hida, K. et al.: Syringo-subarachnoid shunt for syringomyelia using partial hemilaminectomy. Br. J. Neurosurg 1999; 13: 41-45.

9. Kaden, B., Cedich, C., Schultzheiss, R., et al.: Disappearance of syringomyelia following resection of extramedullary lesion. Acta Neurochir 1993; 123: 211-213.

10. Klekamp, J., Batzdorf, U., Samii, M., Bothe, H.W.: Treatment of siryngomyelia associated with arachnoid scarring caused by arachnoiditis or trauma. J Neurosurg 1997; 86: 233-240.

11. Malluci, C. L., Stacey, R. J., Miles, J. B., Williams, B.: Idiopathic siryngomyelia and the importance of occult arachnoid webs, pouches and cysts. Br J Neurosurg 1997; 11: 306-309.

12. Milhorat, T.: Classification of syringomyelia. Neurosurg Focus 2000; 8: Article 1, 1-6.
2008; 19: 556-561

13. Paramore, C.: Dorsal arachnoid web with spinal cord compression: variant of an arachnoid cyst? J Neurosurg (Spine 2) 2000; 93: 287-290.

14. Pesudo, P.V., Llácer, J.L., Talamantes, F., et al: Tratamiento quirúrgico de la siringomielia mediante derivación siringo-subaracnoidea. Neurocirugía 1996. 7: 199-207.

15. Sarabia, R., Sandoval, H., Lobato, R.D., et al.: Estudio comparativo de los resultados quirúrgicos y cambios en resonancia magnética en 29 pacientes con siringomielia. Neurocirugía 1994. 5: 115-120.

16. Sgouros, S., Williams, B.: A critical appraisal of drainage in syringomyelia. J. Neurosurg 1995; 82: 1-10.

17. Tator, C.H., Meguro, K., Rowed, D.W.: Favourable results with syringosubarachnoid shunts for treatment of syringomyelia. J. Neurosurg 1982; 56: 517-523.

18. Vaquero, J., Arias, A., Martínez, R., Aragonés, P.: Experiencia anatomo-clínica en pacientes con siringomielia tratados mediante derivación siringosubaracnoidea. Neurocirugía 1991. 2: 9-16.

19. Williams, B., Page, N.: Surgical treatment of syringomyelia with syringopleural shunting. Br J Neurosurg 1987; 1: $63-80$.

20. Williams, B.: Syringomyelia. Neurosurg Clin North Am 1990. 1: 635-685. 21.Yu Y.L., Moseley I.F.: Syringomyelia and cervical spondylosis: a clinicorradiological investigation. Neuorradiology 1987; 29: 143-151.

Horcajadas, A.; Román, A.; Olivares, G.; Saura, E.; Jorques, A.; Iáñez, B.; Pastor, J.; Martín, J.M.: Siringomielia "idiopática": a propósito de un caso. Neurocirugía 2008; 19: 556-561.

Correspondencia postal: A. Horcajadas Almansa. Cuesta del Pino 65, Casa 29. 18008 Granada. 